\title{
Tracking of Noncooperative Airborne Targets Using ADS-B Signal and Radar Sensing
}

\author{
Ming-Shih Huang, ${ }^{1}$ Ram M. Narayanan, ${ }^{1}$ Yan Zhang, ${ }^{2}$ and Arthur Feinberg ${ }^{3}$ \\ ${ }^{1}$ Department of Electrical Engineering, The Pennsylvania State University, University Park, PA 16802, USA \\ ${ }^{2}$ School of Electrical \& Computer Engineering, University of Oklahoma, Norman, OK 73109, USA \\ ${ }^{3}$ Intelligent Automation, Inc., Rockville, MD 20855, USA
}

Correspondence should be addressed to Ram M. Narayanan; ram@ee.psu.edu

Received 31 October 2012; Revised 2 January 2013; Accepted 6 January 2013

Academic Editor: Srinivas R. Vadali

Copyright (C) 2013 Ming-Shih Huang et al. This is an open access article distributed under the Creative Commons Attribution License, which permits unrestricted use, distribution, and reproduction in any medium, provided the original work is properly cited.

As the Automatic Dependent Surveillance-Broadcast (ADS-B) system has gained wide acceptance, additional exploitations of the radioed satellite-based information are topics of current interest. One such opportunity includes the augmentation of the communication ADS-B signal with a random biphase modulation for concurrent use as a radar signal. This paper addresses the formulation and analysis of a suitable noncooperative multitarget tracking method for the ADS-B radar system using radar ranging techniques and particle filter algorithms. In addition, the low-update-rate measurement due to the ADS-B system specification is discussed in order to provide acceptable estimation results. Simulation results show satisfactory tracking capability up to several kilometers with acceptable accuracy.

\section{Introduction}

During the past half century, air traffic management (ATM) has relied heavily on centralized ground stations using primary surveillance radar (PSR) and secondary surveillance radar (SSR) systems. With dramatic increase in the number of aircraft in the future, PSR and SSR will soon reach their limitations in their capability to monitor aerospace traffic patterns and aircraft locations in a timely manner to avoid collisions. Several decentralized approaches have drawn more attention lately thanks to their potential for quicker response and self-reliance. Independent of air traffic control (ATC), the widely used traffic alert and collision avoidance system (TCAS) monitors the airspace around an aircraft and communicates with other aircraft equipped with a corresponding transponder. TCAS is effective and successful in Class A, $\mathrm{B}$, and $\mathrm{C}$ airspaces and Class $\mathrm{E}$ airspace above 10,000 feet, since aircraft in this airspace are required to be equipped with altitude-reporting transponders. However, TCAS is ineffective for noncooperative aircraft and also in lowaltitude Class $\mathrm{E}$ and uncontrolled airspace. To enable the transformation of the ATM to a new paradigm that can meet the demand for the next 20 years and beyond, several developmental programs are underway, such as Single European Sky Air traffic Research System (SESAR) in Europe [1], Next Generation Air Transportation System (NextGen) in U.S.A. [2], and Collaborative Actions for Renovation of Air Traffic Systems (CARATS) in Japan [3]. The philosophy is to move away from legacy ground based technologies to a new and more dynamic satellite based technology. A key element of SESAR and NextGen is ADS-B, which uses the Global Navigation Satellite System (GNSS) signals to provide air traffic controllers and pilots with precise position information in space, in contrast to the traditional surveillance radar derived data [4]. Aircraft transponders receive GNSS signals and use them to determine the aircraft's precise location in the sky, which is combined with other relevant data and broadcast to other aircraft and air traffic control facilities via a digital data link. Besides ADS-B's wide acceptance in Europe and USA, NAV CANADA commenced operational application of ADS-B as a means of providing aircraft surveillance information to Air Traffic Controllers (ATC) [5], and AirServices Australia commissioned the ADS-B Upper Airspace Project (UAP), providing ADS-B 
coverage across the whole continent [6]. In addition, ADS-B is being used as the key solution for Unmanned Aerial System (UAS) integration in the National Airspace (NAS). When properly equipped with $\mathrm{ADS}-\mathrm{B}$, both pilots and controllers will see the same real-time displays of air traffic, substantially improving safety and minimizing collision probability.

However, before the ADS-B implementation and operation are fully complete, there will be a transition period involving coexistence of ADS-B equipped and nonequipped aircraft. Similar to TCAS, the ADS-B system works only for cooperative aircraft equipped with a corresponding system and is, thus, blind to noncooperative targets that are not similarly equipped. It has been pointed out that the limited use of ADS-B as the sole means of surveillance may lead to a reduction of the integrity of the entire ATC system $[7,8]$. Furthermore, localized problems, such as lack of connectivity to the required four visible satellites, will confuse not only aircraft pilots but also ATC $[9,10]$. Hence, it is desired to find a way to cope with the noncooperative targets while retaining the benefits of the ADS-B system.

Since the emergence of ADS-B concept, some researchers have considered the utilization of existing and installed infrastructure of the surveillance radar to combine with the satellite-based ADS-B system within the perspective of ATC. More interestingly, the use of the ADS-B signal itself to detect noncooperative targets from the ADS-B message and from the radar processing, as an onboard collision avoidance system, was first described in a patent disclosure [11], and the concept subsequently developed further [12-15]. The novelty of the ADS-B radar system lies in that the system insightfully exploits the ADS-B out signal, which is primarily designed for communication purposes, as a radar signal to perform multiple target estimation and tracking, thereby creating a multifunctional waveform. However, details of such radar-communication system specifications and problems of low-update-rate measurement due to the ADS-B system requirement have not been fully discussed. This paper provides details of the ADS-B radar system design, including the link power budget, and RF signal interference analysis. Furthermore, multitarget tracking using particle filter for the ADS-B radar system is proposed. In addition, the slow convergence in particle filter algorithm and the measurement at low-update-rate, owing to the ADS-B signal broadcast rate of once per second, is addressed. It is important to note that in the ADS-B radar system, the radar capability is independent of the ADS-B message data integrity. Therefore, the coexistence of ADS-B and radar sensing achieves increased reliability and redundancy, because it becomes possible to identify malfunctioning sensors and removes false alarms through performing conformance checks by comparing communication and radar reports.

The remainder of the paper is organized as follows. Section 2 briefly reviews the evolution of air surveillance systems. The ADS-B radar system architecture and link budget are provided in Section 3. Then, the multitarget estimation algorithm for ADS-B radar system is proposed and discussed in Section 4. Computer simulations that demonstrate the functionality of ADS-B radar system for multitarget estimation performance are provided in Section 5. Conclusions are drawn in Section 6.

\section{Evolution of Surveillance Technologies}

For the sake of completeness and continuity, a brief and comparative review of the existing and new surveillance technologies is provided in this section. The major advantages and disadvantages for each approach are pointed out with an emphasis on the noncooperative surveillance capability. In addition, it can also be seen through the transition of the surveillance technologies that ATM is shifting from centralized systems to decentralized systems as the density of the air traffic continues to increase.

2.1. PSR and SSR. For the past few decades, PSR and SSR have been the main two components of an ATC station. PSR has the capability to detect large metal objects, including cooperative and noncooperative targets, while SSR works only for transponder-equipped aircraft. SSR relies on aircraft with corresponding transponder, but the report provides aircraft identification. Both PSR and SSR were designed for low and medium traffic situations. Due to the insufficient range ambiguity, they are unable to meet the challenge of high traffic volume. Moreover, ground-based surveillance radar has limitations at low altitudes and may be influenced by atmospheric conditions.

2.2. TCAS. Due to continuing growth in air traffic, TCAS or other similar devices have been in various stages of research and development since the early-to mid-1950s to serve as a last resort collision avoidance safety net. TCAS operates similarly to the ground-based SSR but independently interrogates surrounding aircraft on a $1030-\mathrm{MHz}$ radio channel. The pilot will be alerted to the presence of the intruding aircraft replying to the interrogation via $1090-\mathrm{MHz}$ radio frequency. Current generation TCAS II, jointly developed by the US Radio Technical Commission for Aeronautics (DO-185B) and European Organization for Civil Aviation Equipment (ED143), issues two types of advisories: the resolution advisory (RA), which identifies an intruder that is considered a collision threat, and the traffic advisory (TA), which identifies an intruder that may soon cause an RA. According to the predicted closest point of approach (CPA), TCAS produces a TA at approximately 45 seconds and an RA at 35 seconds to CPA. TCAS is designed to reduce the incidence of mid-air collisions and has been very successful since its introduction. However, the major concern with regard to either TCAS or ADS-B is that they are not required all the time, and ADS-B out is not yet mandated in most countries. Aircraft equipped with TCAS and/or ADS-B are still exposed to danger of collisions in low altitude of Class $\mathrm{E}$ and uncontrolled airspace owing to their inability to detect noncooperative targets and unawareness of any illegal intruder in transponder-required airspace.

2.3. ADS-B. ADS-B is redefining the paradigm of communication, navigation, and surveillance in ATM. An ADS-B equipped aircraft determines its own position using GNSS 


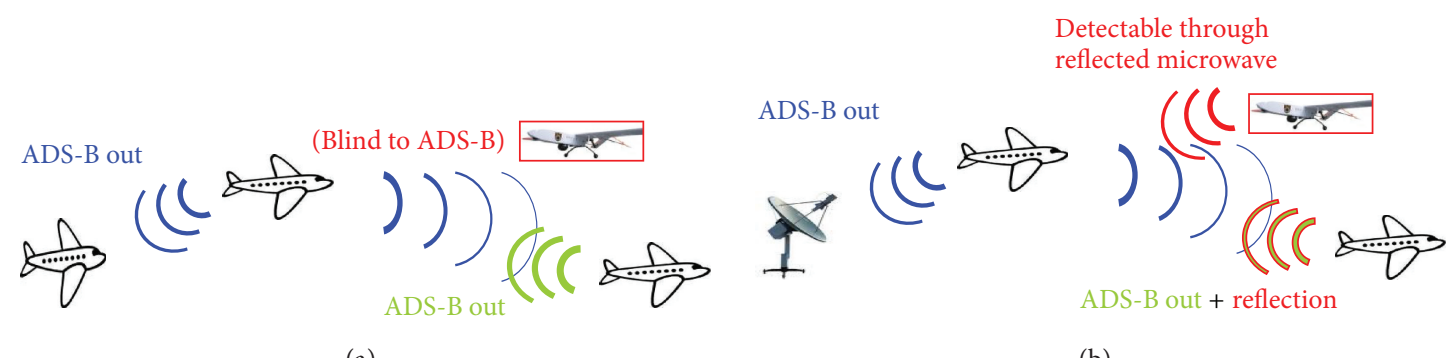

(a)

(b)

FIGURE 1: Surveillance principles and concepts for ADS-B and ADS-B radar. (a) ADS-B: communication between equipped aircraft and ATC. (b) ADS-B radar: able to detect both equipped and nonequipped aircraft.

and periodically broadcasts its four-dimensional position (latitude, longitude, altitude, and time), track and ground speed, aircraft or vehicle identification, and other additional relevant data as appropriate, for example, intended trajectories [16], to nearby aircraft also equipped with the ADS-B system and potential ground stations without expectation of an acknowledgement or reply. One of the most significant advantages of the ADS-B system is that it minimizes radio frequency (RF) spectral congestion as would be generated by TCAS. Any user, either aircraft or ground stations within broadcasting range, may receive and process ADS-B surveillance information. ADS-B system provides accurate information and improves situational awareness. Moreover, ADS-B enables a shift from a centralized, ground-based ATM system to a decentralized network involving pilots and aeronautical operational control centers. It might eventually allow pilots to use onboard instruments and electronics to maintain a safe separation and to reduce their reliance on ground controllers.

2.4. ADS-B Radar. While the ADS-B system can only be considered as a decentralized cooperative surveillance ATM technique, the ADS-B radar system deals with both cooperative and noncooperative targets. Figure 1 depicts the general working principle for ADS-B radar concept, as well as the comparison between the ADS-B system and the ADS-B radar system. Noncooperative targets, such as unmanned aerial vehicles (UAVs) and private jets, are blind to the ADS-B systems when not equipped with the ADS-B system, but they pose equal collision danger if not being detected. Moreover, an inexpensive backup surveillance is necessary in the case of lost, incorrect, jammed, or compromised satellite information possibly caused by localized problem or device malfunction. The ADS-B radar system is introduced as an insightful modification to the standard ADS-B system in the interests of a safe backup service without significantly enlarging the volume of ADS-B equipment. Since the ADS$B$ systems broadcast signals periodically, the reflective ADS$B$ electromagnetic energy could be viewed and extended for use as radar echoes. The returned echoes will be exploited to detect and track targets, including noncooperative ones, and hence, the pilot can be alerted without relying on guidance from the ground station to maintain aviation safety. Furthermore, by comparing the communication and radar reports

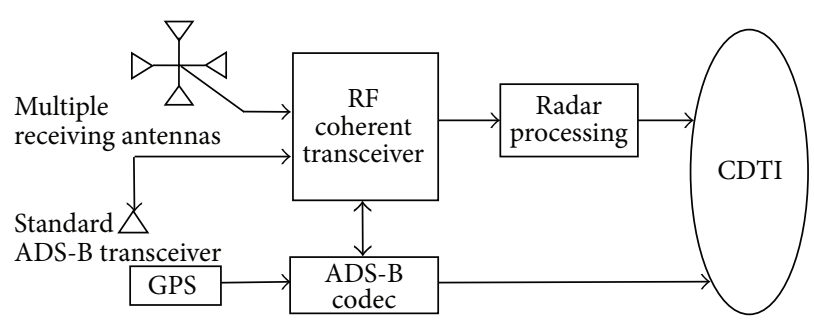

Figure 2: Conceptual architecture of ADS-B radar system.

for the ADS-B equipped targets, the ADS-B radar system can suppress the creation of tracks based on ADS-B messages that contain intentionally incorrect position information.

\section{System Design Specification}

A conceptual architecture of the ADS-B radar system is depicted in Figure 2. This differs from the corresponding figure in Zhang and Qiao [11] by pointing out the possibility to fuse the information from the radar processing and the ADS-B message, as well as the target estimation method in radar processing. The fundamental principle of the ADS- $\mathrm{B}$ radar system is to use reflective ADS-B signals with only four additional L-band antennas on the standard ADS-B system to perform multitarget estimation and tracking for both cooperative and noncooperative targets. Because the ADS$B$ receivers only respond to the envelope of the incoming signal, phase modulation will not affect the normal ADS-B signal decoding for other aircraft. As a result, the standard ADS-B system will still be able to correctly decode the phasemodulated signal to obtain the communication message. The ADS-B radar system retains the communication capability and receives the standard ADS-B messages from neighboring aircraft, just as the standard ADS-B system does. Once per second, it also broadcasts the phase modulated ADSB signal. After sending out the electromagnetic wave, the receiving antennas capture the returned signal reflected from surrounding metallic objects, and cross-correlation is performed between the transmitted burst and the returned echoes. Both the ADS-B In information and the estimated location from the radar report can be fed into Cockpit Display of Traffic Information (CDTI) [17]. 


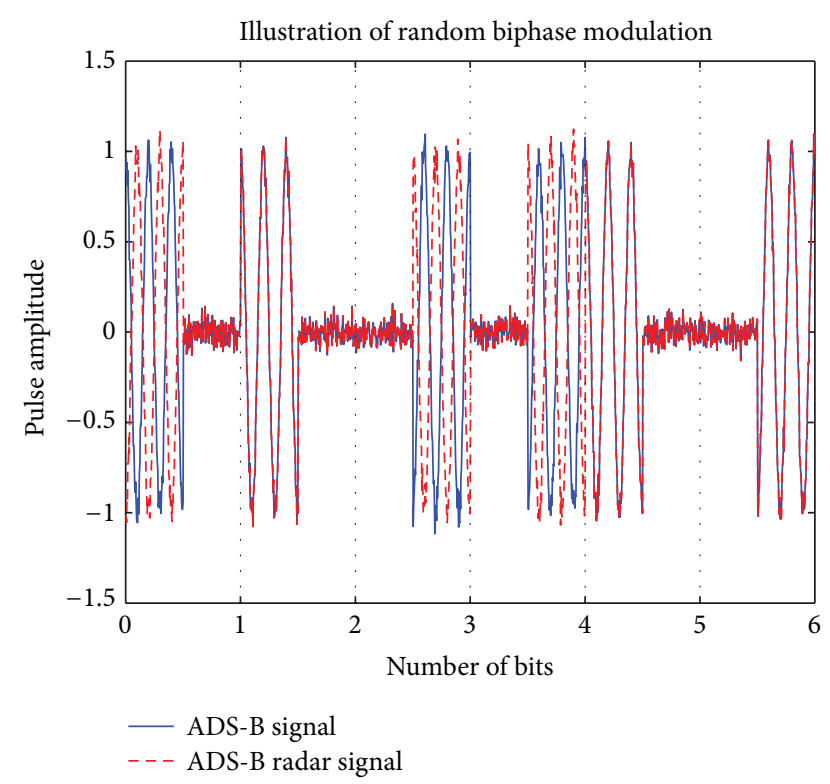

FIGURE 3: Illustration of the ADS-B waveform (blue) versus the ADS-B radar waveform (red).

The design consideration is focused on the feasibility of utilizing the returned ADS-B signal as a radar echo in terms of the signal-to-noise ratio and radar performance. In addition, the $500 \mathrm{~W}$ peak transmit power, the $120 \mu$ s pulse duration specifications of the $1090 \mathrm{MHz}$ ADS-B standard, and the broadcasting rate impose constraints on the system design, requiring a detailed analysis.

3.1. Signal Waveform and Interference Analysis. Each of the ADS-B Extended Squitter is $120 \mu \mathrm{s}$ long, including $8 \mu \mathrm{s}$ preamble and $112 \mu$ s data block, and is similar to TCAS message format, except for longer $56 \mu$ s ADS information. Besides, the ADS-B message is digitally encoded using pulse position modulation (PPM). When a communication signal is utilized and treated as a radar signal, many issues need to be addressed. First, the signal must be suitably modulated for performing matched filtering in the receiver. The product of the PPM signal waveforms for two different bits can only be either zero or positive, which translates into a raised correlation noise floor. Second, the constraints of pulse duration, peak transmit power, signal bandwidth, and coherent returned signals degrade the radar performance. Therefore, the primary task for the ADS-B radar design is to exam the feasibility of treating the ADS-B signal as a radar signal.

In order to improve the radar performance, the major modification to the ADS-B signal is random biphase modulation introduced to each bit. Figure 3 shows the simulated ADS-B waveform and the proposed ADS-B radar waveform. Both signals are of the same duration and have identical digital messages, except that the ADS-B radar signal has a random $180^{\circ}$ phase shift. By randomizing the transmit signal, the matched filtering operation can be performed by cross-correlating the reflected signal with a time-delayed replica of the transmit waveform. The biphase modulation renders both positive and negative products, forcing the

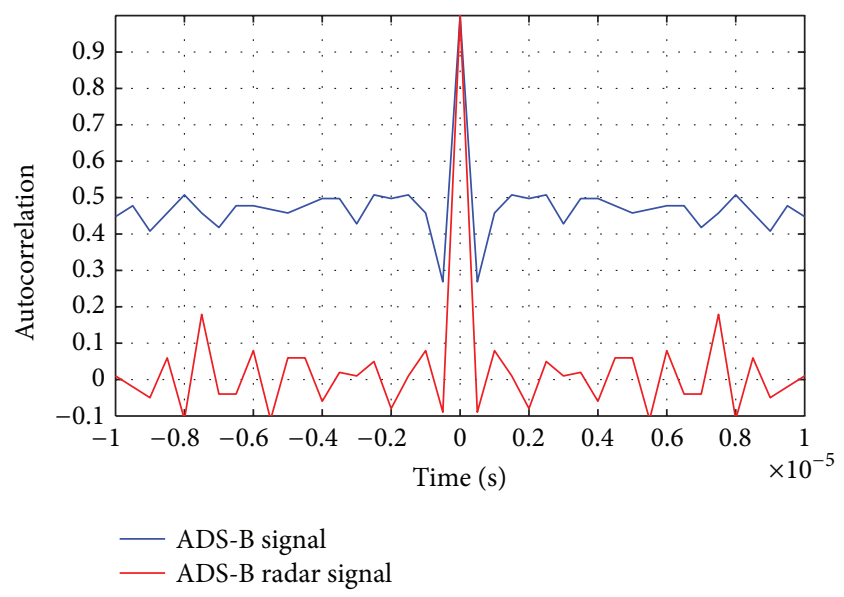

FIGURE 4: Autocorrelation of standard ADS-B signal and autocorrelation of the ADS-B radar signal.

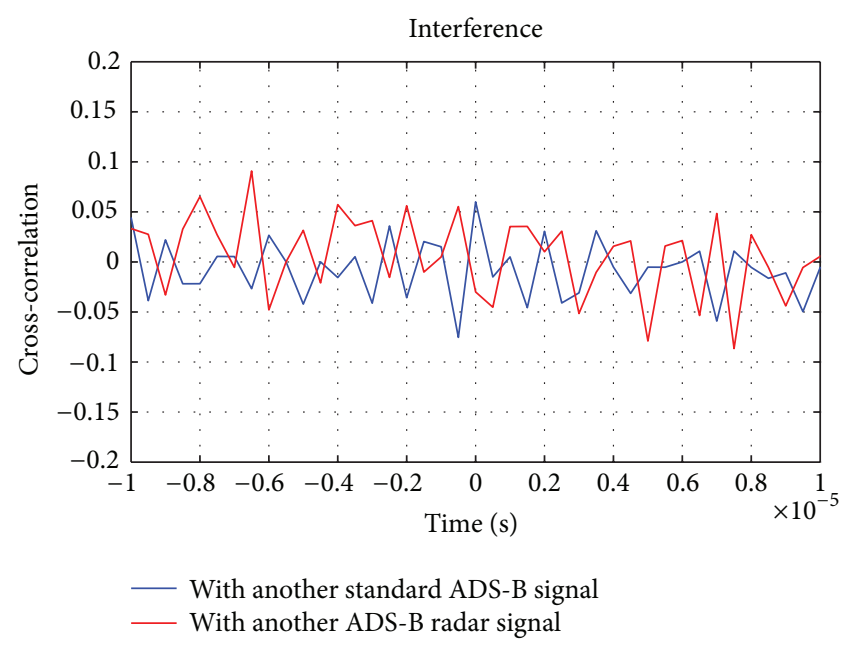

FIGURE 5: Cross-correlation of a standard phase-modulated ADS$B$ signal with another standard ADS-B signal and another phasemodulated ADS-B radar signal.

autocorrelation to be statistically zero except for zero timelag. The effect of the sum of the products in the autocorrelation function is provided in Table 1 . The significance of the random biphase modulation $\left(0^{\circ}\right.$ or $\left.180^{\circ}\right)$ can be seen clearly in the autocorrelation of the ADS-B radar signal and the ADS-B signal, as depicted in Figure 4. It is clear that the autocorrelation of the ADS-B radar signal outperforms that of the original modulation-free ADS-B signal and shows an improved correlation peak-to-noise ratio.

Since it is very likely to have other ADS-B signals in the same channel, it is critical to analyze the interference of the ADS-B signals and possibly other ADS-B radar signals as well. Figure 5 shows that a typical ADS-B radar signal is uncorrelated with both a standard ADS-B signal as well as another independent ADS-B radar signal, thereby indicating that the on-board ADS-B radar receiver will not be affected by standard ADS-B or ADS-B radar transmissions from other aircraft in the vicinity. 
TABLE 1: Effects of random biphase modulation on correlation results.

\begin{tabular}{lccc}
\hline \multicolumn{4}{c}{ Modulation } \\
Messages & PPM & \multicolumn{2}{c}{ PPM with random biphase PM } \\
& & Same phase & Inverted phase \\
& & (no phase shift $)$ & $\left(180^{\circ}\right.$ phase shift $)$ \\
\hline \multirow{2}{*}{$(1,1)$ or $(0,0)$} & Positive & Positive & Negative \\
& sum-product & sum-product & sum-product \\
$(1,0)$ or $(0,1)$ & Zero & Zero & Zero \\
& sum-product & sum-product & sum-product \\
\hline
\end{tabular}

Because the modulated signal has wider bandwidth (the resultant waveform still complies with the ADS-B standard), the range resolution is improved and the range side lobes are also further suppressed [18]. The range resolution of 75 meters for the ADS-B radar system is sufficient for most collision avoidance applications.

3.2. System Configuration and Link Budget Analysis. The ADS-B radar system is composed of the following components: (a) ADS-B transceiver, (b) ADS-B encoder/decoder, (c) radio frequency (RF) electronics with up- and downfrequency conversion, crosstalk cancellation, and filtering capabilities, (d) a phase modulator, and (e) four additional antennas operating at $1090 \mathrm{MHz}$. In particular, (d) and (e) are additional to the original ADS-B system to process the reflected radar signals and to estimate target locations.

The link power budget is analyzed based on the radar range equation as follows:

$$
P_{r}=\frac{P_{t} G_{t} G_{r} \lambda^{2} \sigma}{(4 \pi)^{3} R^{4}}
$$

where $P_{r}$ and $P_{t}$ are the received and transmitted power, respectively; $G_{t}$ and $G_{r}$ are the receiving and transmitting antennas gain, respectively; $\lambda$ is the signal wavelength; $\sigma$ is the target's radar cross-section (RCS); $R$ is the range to the target. To improve range resolution, common radar signal pulse durations are as short as tens of nanoseconds. However, since the signal energy is emitted continuously, integration gain can be achieved by utilizing the 545 sinusoidal cycles in each bit. As shown in Table 2, for a target having an RCS of $0 \mathrm{dBsm}$ (1 square meter) at a distance of $4.5 \mathrm{~km}$, the signalto-noise ratio (SNR) is $5.0 \mathrm{~dB}$, a desirable value. For a large airliner with a higher RCS of $20 \mathrm{dBsm}$ (100 square meters), the operational range for the same SNR value could extend to $14 \mathrm{~km}$, which allows more than one minute of reaction time.

\section{Multitarget Estimation and Tracking Algorithm}

The Multiple Signal Classification (MUSIC) technique [19] was initially employed for the ADS-B radar to perform detection and estimation of single target angle-of-arrival (AOA). Due to the nature of the ADS-B radar signal, there are several important factors that limit the extension to multitarget estimation, primarily (a) the number of detectable targets and (b) signal coherency. The MUSIC algorithm has the well-known inherent constraint that the number of the receivers has to be larger than that of the targets. With fixed number of $N$ antennas, the maximum number of detectable targets using the MUSIC algorithm is $N-1$. While it is desirable to minimize the cost and amount of antennas used in the ADS-B radar system, the detection capability becomes strictly limited. To further elaborate the signal coherency problem, the returned signals bouncing off from different targets are highly correlated because all the reflected radar echoes are simply scaled replicas of the transmitted signal but slightly frequency shifted by the Doppler shift, which is about $1 \mathrm{kHz}$ for the relative speed of $150 \mathrm{~m} / \mathrm{s}$. Although there has been research dealing with correlated signals, such as constrained MUSIC [20], cumulant-based coherent signal subspace method [21], and focusing matrix for coherent signal subspace processing (for wide-band signals) [22], these approaches do not apply to the scenario that the ADS-B radar system encounters owing to either unavailable prior information or limited signal bandwidth from the ADS-B signal specification. Other methods, like the least squares and the unscented Kalman filter approaches, require prior information of the motion model. A hybrid approach that combines the time-of-arrival (TOA) and the particle filter approaches for multitarget estimation and tracking for the ADS-B radar system is proposed in this paper. In the particle filter algorithm, the unavailable target motion can be dealt with using the concept of jittering [23], by introducing a noise model to cover the uncertainty of the target location. After the radar report is obtained, multisensor data fusion is a discrete problem, and attempts to combine ADS-B messages with radar reports are described in $[7,8]$.

4.1. Multitarget Detection and Ranging through TOA. Let $s(t)$ represent the transmitted signal. The distance between the target and the own aircraft can be obtained by crosscorrelating the delayed transmitted signal, $s\left(t-\tau_{d}\right)$, with the received signal, $s\left(t-\tau_{r}\right)$, where $\tau_{d}$ is the internal delay and $\tau_{r}=2 R / c$ is the round-trip time to the target. The target range is $R$, and the speed of light is $c$. In theory, the crosscorrelation peak occurs when $\tau_{d}=\tau_{r}$, from which the range can be determined. In our simulation experiment, the peak location may vary a little due to the measurement noise and phase coding scheme. In the ADS-B radar system, $s(t)$ is the phase-modulated ADS-B signal. By continuously crosscorrelating the received signal with the delayed transmitted signal replicas, the number of peaks above a predetermined threshold indicates the number of detected targets. Moreover, the ranges can be calculated based on the delay times as described above. To correctly associate the ranges with the correct targets can be a difficult problem [24, 25]. However, because the sensors are located only several meters apart, the returned echoes from different targets usually arrive at the sensors in the same sequence.

4.2. Multitarget Estimation. The particle filter algorithm is briefly reviewed in this section. In addition, a simple multitarget estimation method based on particle filter is proposed for the application of the ADS-B radar system. 
TABLE 2: Link budget analysis.

\begin{tabular}{|c|c|c|c|}
\hline \multicolumn{2}{|c|}{ Receiver noise floor $=-110.9 \mathrm{dBm}$} & \multicolumn{2}{|c|}{ Received signal power $=-105.9 \mathrm{dBm}$} \\
\hline Noise figure & $3 \mathrm{~dB}$ & Peak transmit power & $+57 \mathrm{dBm}$ \\
\hline \multirow[t]{6}{*}{ Bandwidth } & $1 \mathrm{MHz}$ baseband & $\begin{array}{c}\text { Processing gain } \\
\text { (545 samples coherently integrated) }\end{array}$ & $27.4 \mathrm{~dB}$ \\
\hline & & $\begin{array}{c}\text { Antenna gain } \\
\text { (omnidirectional) }\end{array}$ & $0 \mathrm{~dB}$ \\
\hline & & Assumed RCS & $0 \mathrm{dBsm}$ \\
\hline & & $1 /(4 \pi)^{3}$ & $-33.0 \mathrm{~dB}$ \\
\hline & & $1 /(\text { Range })^{4}$ (at $\left.4.5 \mathrm{~km}\right)$ & $-146.1 \mathrm{dBm}^{-4}$ \\
\hline & & Square of wavelength & $-11.2 \mathrm{dBsm}$ \\
\hline & & io, $\mathrm{SNR}=5.0 \mathrm{~dB}$ & \\
\hline
\end{tabular}

Furthermore, the problem of slow convergence in particle filter and measurements at low-update-rate is addressed.

4.2.1. Particle Filter Algorithm. Particle filter [26-28], also known as sequential Monte Carlo method, bootstrap filtering [29], and the condensation algorithm in computer vision [30], is very suitable for nonlinear and nonGaussian estimation and tracking applications as encountered in the real world. A particle filter is essentially composed of three stages: prediction, update, and resampling. The prediction stage uses the system model to predict the state probability density function (PDF) forward from one measurement time to the next. Since the state is usually subject to unknown disturbances, prediction generally spreads the state PDF. The update operation takes the latest measurement to modify the prediction PDF using Bayes' formula. A resampling step was introduced by Gordon et al. [31] in order to discard the particles with very low weights. Particle filters work by providing a Monte Carlo approximation to the PDF which can be easily updated to incorporate new information as it arrives.

A discrete time estimation problem is considered here. The state vector is denoted by $\mathbf{x}_{t}$ whose temporal evolution is given by the state equation:

$$
\mathbf{x}_{t}=\mathbf{f}\left(\mathbf{x}_{t-1}\right)+\mathbf{v}_{t-1}
$$

where $\mathbf{f}$ is the state transition function, and $\mathbf{v}_{t}$ is the process noise with zero mean but not necessarily having a Gaussian distribution. In the ADS-B radar system, the components of the state vector will be target locations. At each discrete time point an observation $\mathbf{y}_{t}$, related to the state vector, can be represented as follows:

$$
\mathbf{y}_{t}=\mathbf{h}\left(\mathbf{x}_{t}\right)+\mathbf{n}_{t}
$$

where $\mathbf{h}$ is the measurement function and $\mathbf{n}_{t}$ is the measurement noise with zero mean but not necessarily having a Gaussian distribution. $\mathbf{n}_{t}$ is assumed to be uncorrelated with $\mathbf{v}_{t}$. In our application, $\mathbf{y}_{t}$ is the range information and $\mathbf{h}$ is the process to obtain the target ranges through the TOA technique.

A numerical integration method for the particle filter algorithm is listed below.
(1) Particle generation: within the field of view (FOV), create $N$ particles and associated weights $\left(\mathbf{x}_{t-1}^{n}, w\left(\mathbf{x}_{t-1}^{n}\right)\right)_{n=1, \ldots, N}$ according to the uniform distribution.

(2) Prediction: particles propagate according to evolution equation (1).

(3) Measurement update: use the available measurements to compute the likelihood of each particle and update the weights of all particles with a posteriori density. This is accomplished using

$$
w\left(\mathbf{x}_{t}^{i}\right) \propto w\left(\mathbf{x}_{t-1}^{i}\right) p\left(\mathbf{y}_{t} \mid \mathbf{x}_{t}^{i}\right)
$$

where the final weights sum to one, namely, $\sum_{i=1}^{N} w\left(\mathbf{x}_{t}^{i}\right)=1$.

(4) Systematic resampling: after a predetermined number of iterations, take $N$ samples with replacement from the current particle set based on $w\left(\mathbf{x}_{t}^{i}\right)$.

(5) Iteration: letting $t=t+1$, repeat the process until desired estimation error is achieved.

For abruptly changing systems, Interacting Multiple Model (IMM) method [32-34] and Generalized PseudoBayesian (GPB) [35] are widely used in the target tracking literature. To reduce the computational complexity on the ADS-B radar system, these approaches are not adopted. In fact, we do not even use the motion equation, since the dynamic model of the object cannot be obtained. In the prediction step, particles are simply scattered with a large variance that is able to cover abrupt motion changes.

Assume there are two targets around the own aircraft equipped with ADS-B radar system. Each particle will be assigned two weights according to the state PDF. In the resampling step, these two weights will be merged by taking the largest weight for each particle. This process has two-fold implication: (a) the peak selection from the posterior distribution and (b) partition of the particles to represent each target. The index of the maximum value out of the two weights for one particle will be used to mark which target this particle is associated with and is more likely to be close to. Since no two targets should and will share the same location, the larger weight of the partitioned particle is significant 


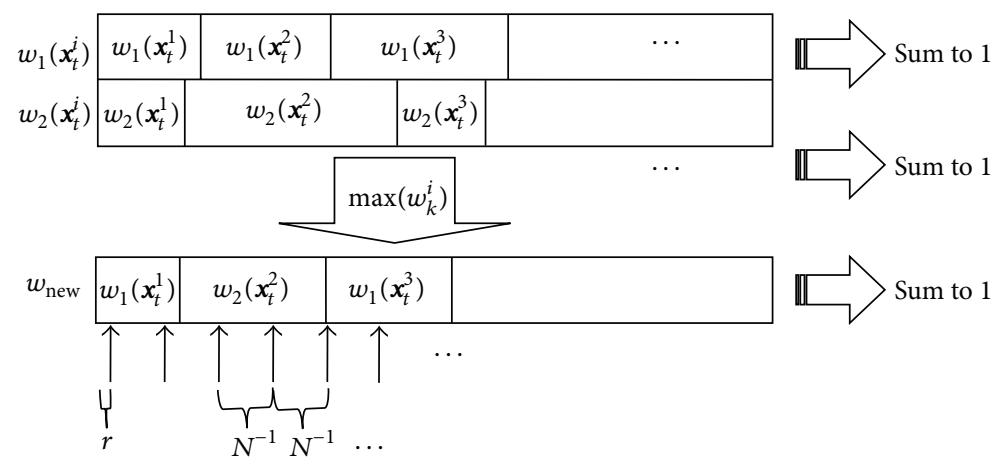

FIGURE 6: Resampling mechanism for multiple targets.

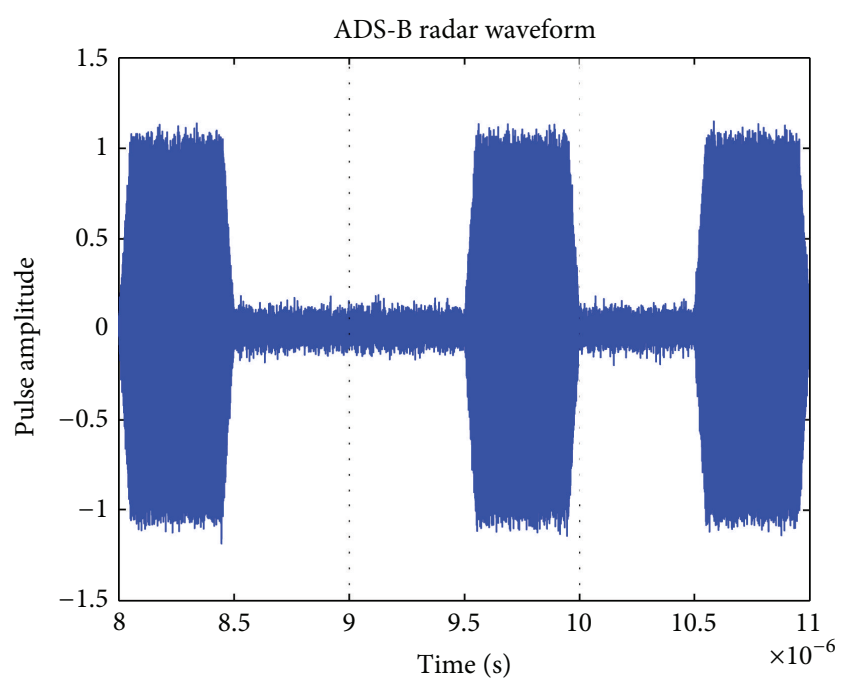

Figure 7: Transmitted ADS-B radar signal waveform.

and retained while the smaller weight is discarded. After the resampling procedure, all particles whose both weights are low will be discarded and only those particles with at least one high weight will survive. Moreover, parts of the particles will be representing Target 1 if their first weights are clearly larger than the second ones. Figure 6 depicts the idea of such resampling mechanism for multiple targets. The resampling step removes particles that are improbable to be any of the targets, but it requires additional processing latency. Hence, we would like to intelligently pick up the right time to do the resampling step. The parameter $N_{\text {eff }}$, the effective sample size, can be used to measure the degeneracy of the algorithm $[36,37]$. Once the particles spread out all over the place and only a certain amount of particles are meaningful, $N_{\text {eff }}$ will become small. This is the right time to spend extracomputational expense to discard low weight particles. A good estimate of the effective sample size is given by $\widetilde{N}_{\text {eff }}=\left[1+N^{-1} \sum_{i=1}^{N}\left[N \times w\left(\mathbf{x}_{t}^{i}\right)-1\right]^{2}\right]^{-1}$, which ranges from $100 \%$ to $0 \%$. The resampling step is performed when the number of meaningful particles is less than a predetermined threshold value, $N_{\text {threshold }}$. This enables the particle filter algorithm to simply do the resampling at appropriate times.
The numerical approximation of adaptive resampling for $m$ targets is given as follows, if $m$ is known.

The procedure is described below.

(1) Set up $N_{\text {threshold }}$, which represents the number of the meaningful particles, to be within $[0,1]$.

(2) Derive $w_{\text {new }}=\left\{\left.\max \left(\mathbf{w}_{k}^{i}\right)\right|_{k=1, \ldots, m}, i=1, \ldots, N\right\}$.

(3) Calculate $\widetilde{N}_{\text {eff }}=\left[1+N^{-1} \sum_{i=1}^{N}\left[N \times \mathbf{w}_{k}^{i}-1\right]^{2}\right]^{-1}, k=$ $1, \ldots, m$.

(4) If $\min \left\{\widetilde{N}_{\text {eff }}\left(w_{1}\right), \ldots, \widetilde{N}_{\text {eff }}\left(w_{m}\right)\right\}<N_{\text {threshold }}$, then take $N$ samples with replacement from the current particle set using $w_{\text {new }}$.

4.2.2. Supplementary Particle Filter Algorithm. As the ADS$B$ radar measurement is available once per second and the PF can be completed in the order of one-tenth of a second, most of the time the system is idling and waiting for the new measurement. To improve the estimation performance, some sort of upsampling process is desirable and can be realized through piecewise constant interpolation between successive measurements. We name the algorithm using interpolated measurement on $\mathrm{PF}$ as the supplementary particle filter (SPF). Before the new measurement arrives, SPF performs iteratively using the current observation as if the target were static. Standard PF is vulnerable to sample impoverishment, because a finite number of particles are used to approximate a continuous distribution. The benefit of SPF over standard $\mathrm{PF}$ is that SPF minimizes the estimation error when the sample size is not sufficiently large. SPF provides improved estimation accuracy because particles will be redistributed to high likelihood areas over iterations although the same measurement information is reused. The sample resolution is essentially enhanced during SPF iterations, thereby improving the estimation performance.

SPF not only improves the estimation accuracy between successive measurements but also benefits further the estimation result when new measurement arrives, because more particles are already allocated to local mode of the posterior density. Through extensive simulation analysis, it has been noticed that PF takes many iterations to converge. While the ADS-B message is available only once a second, a few dozen seconds may be needed to obtain accurate estimated 

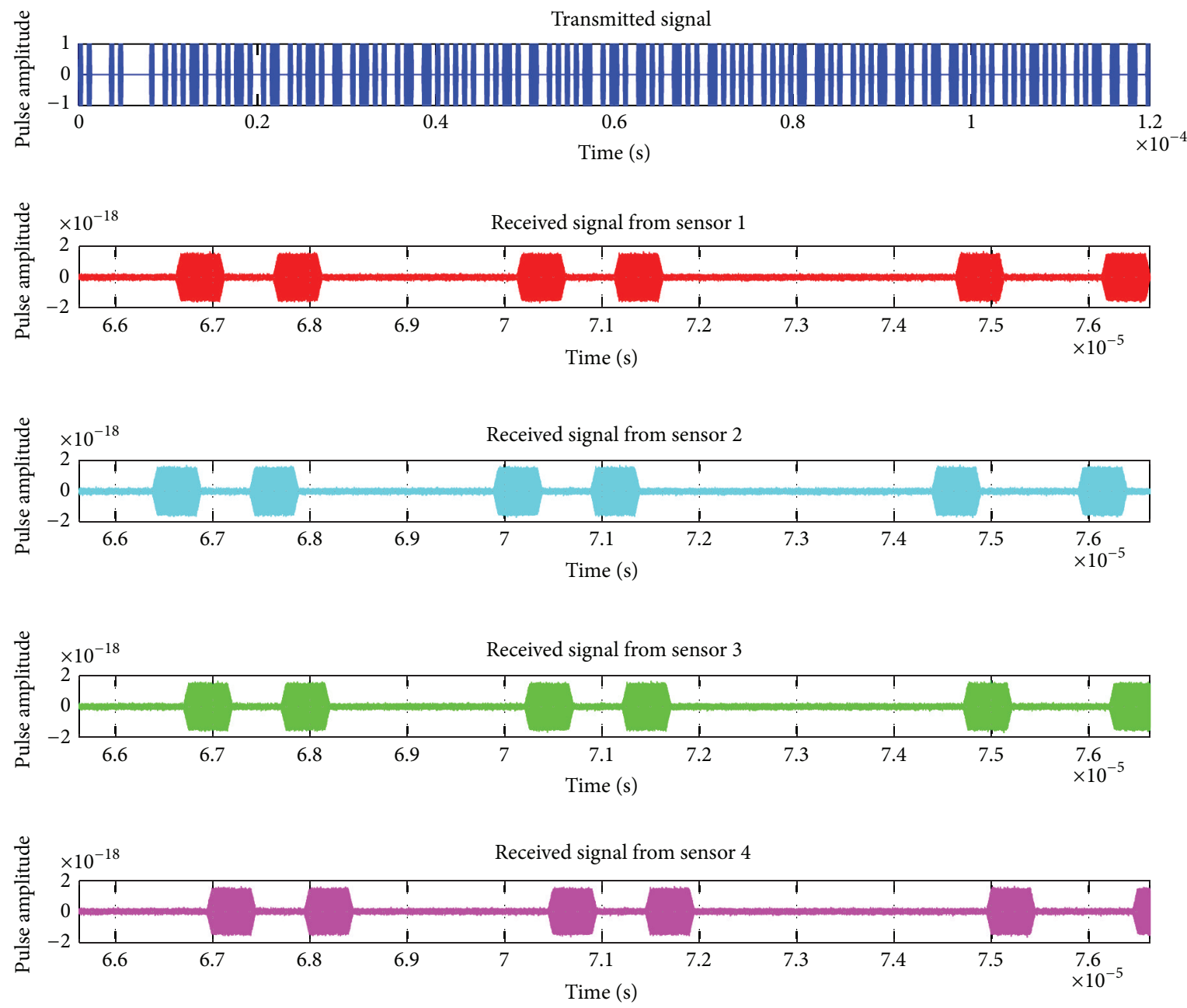

FIgURE 8: Transmitted ADS-B radar signal and received signals from four sensors.

target locations. However, for the pilots to react on imminent collisions, it is critical to improve the convergence rate and estimation accuracy in spite of the low measurement update rate. Overall, the convergence rate of SPF is about 2-3 times faster than PF, and the estimation error of SPF is about onehalf of the estimation error using standard PF. The gain is significant especially during the very first few measurements.

\section{Simulation Results}

In order to ascertain the effectiveness of the algorithm for the ADS-B radar system, the transmitted signal is simulated according to the ADS-B radar message format, which follows the requirements of the ADS-B signal specification. The ADS$B$ radar waveform, as shown in Figure 7, is generated by adding random biphase modulation into each bit of the ADSB signal. The signals received by multiple sensors are essentially the attenuated and delayed replica of the transmitted signal with measurement noise, $\sigma_{n}$. The sensor locations are not restricted, and in the simulation setup, the sensors are circularly positioned and 30 meters apart, using the maximum available distance on an airplane. The transmitted
ADS-B radar signal and the returned signals received by the four sensors are shown in Figure 8. The time differences between the transmitted and received signals are used to calculate the ranges from the target to each of the sensors. Since the ADS-B radar signal is broadcast every second, a new measurement will also arrive approximately once per second.

Without prior estimation of motion model, additional positional uncertainty is incorporated in the state transition equation, as described in Section 4.2.1, to allow the particles to have the potential to move around and to compensate the unknown maneuver while searching for the best solution. As long as one or a few particles are able to "follow" the target, the calculated weights will be high, and subsequently, in the next resampling step, a large amount of particles will be drawn to the neighboring regions of the particles that have high weights. In the simulation, the positional uncertainty is set to $300 \mathrm{~m}$ after considering the relative aircraft speed and the finite amount of particles. By taking into account the detection range of $10 \mathrm{~km}$ and the computational load for multitarget tracking, the number of particles is chosen to be 10,000 and $N_{\text {threshold }}=5 \%$ for the following three simulation scenarios: (1) constant velocity with random acceleration and 


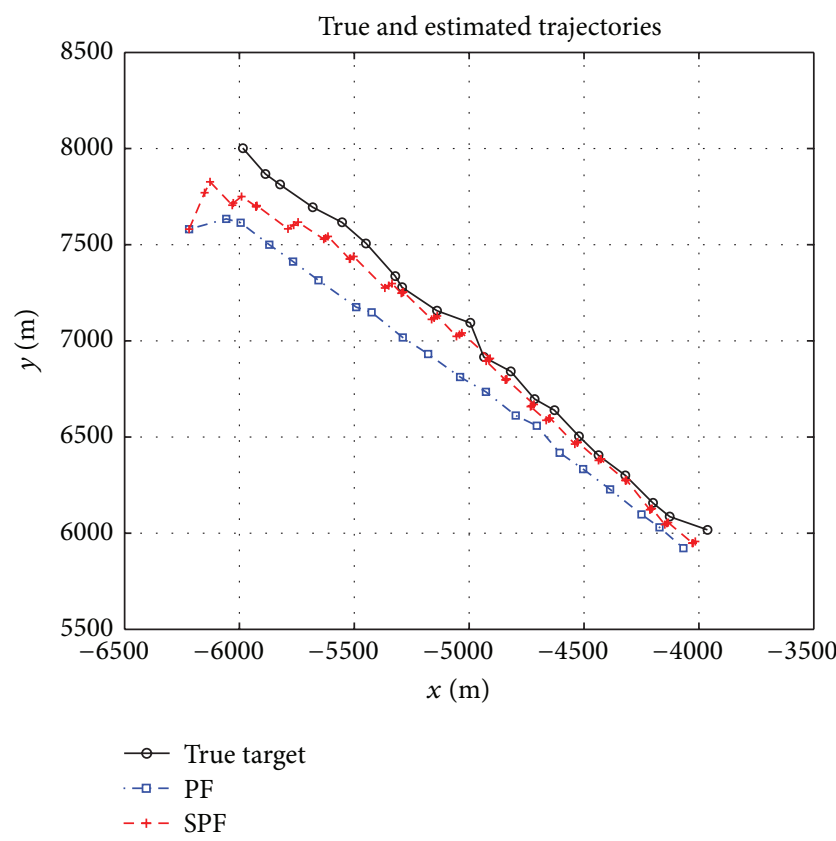

FIGURE 9: Tracking trajectories of PF and SPF methods against true target (20 MC trials).

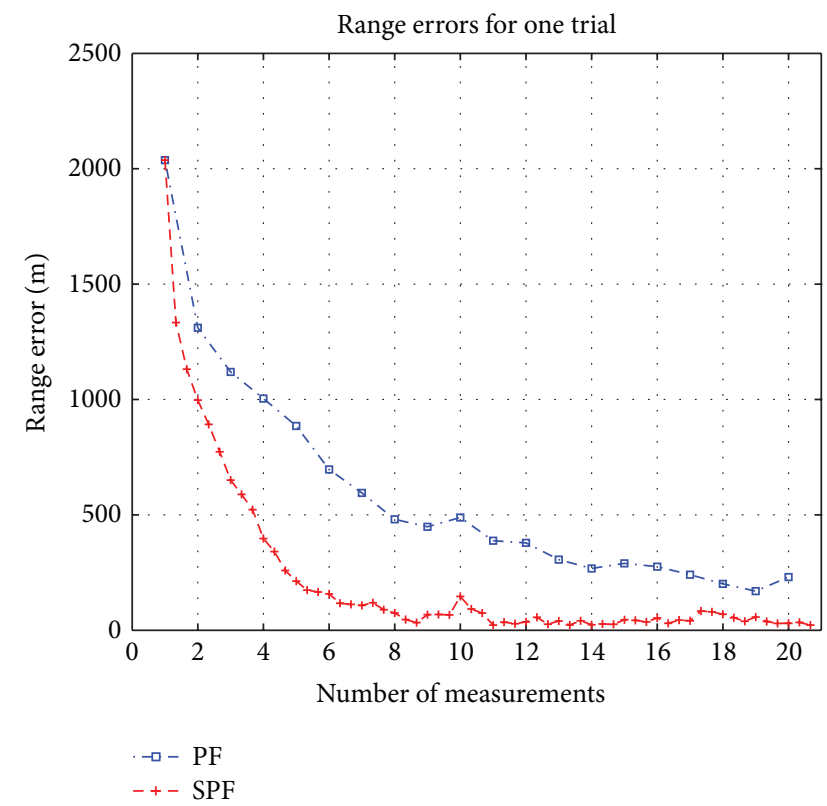

FIGURE 10: Range errors during each iteration (one trial).

direction noise, (2) basic flight maneuvers, and (3) multiple targets.

To test the tracking capability, we first assume a noncooperative aircraft having an RCS of $20 \mathrm{dBsm}$ at a range of $10 \mathrm{~km}$. According to the link budget analysis, as discussed in Section 3.2, the SNR is $11.1 \mathrm{~dB}$. The target is moving at a constant velocity of $150 \mathrm{~m} / \mathrm{s}$ with a Gaussian distributed acceleration and heading direction. Figure 9 depicts the tracking performance comparison of PF and SPF against the

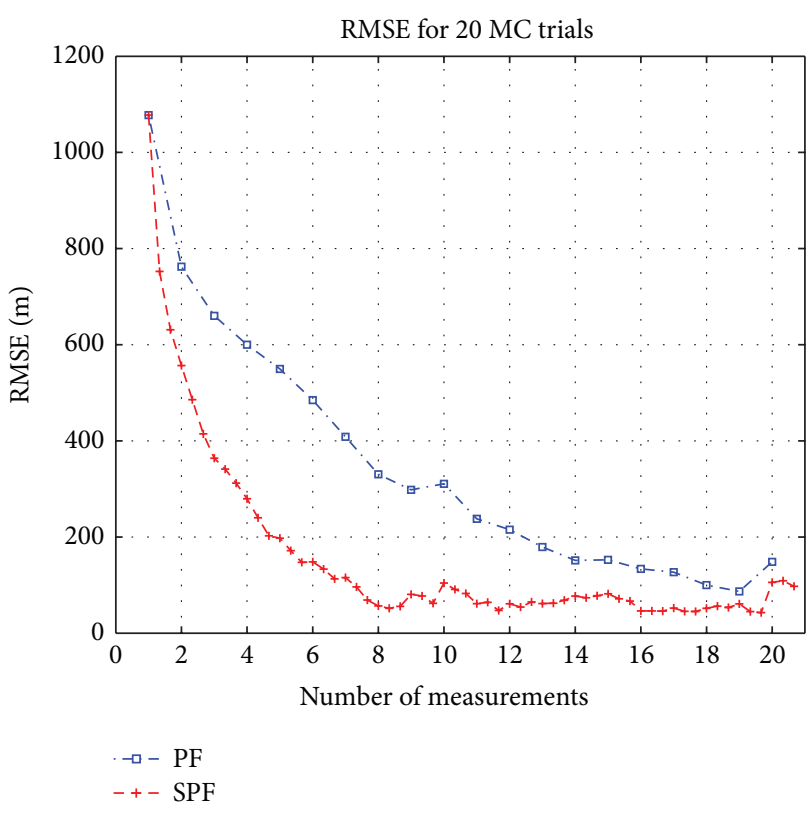

FIGURE 11: RMSE for a target with constant velocity, as well as Gaussian distributed acceleration and heading direction (20 MC trials).

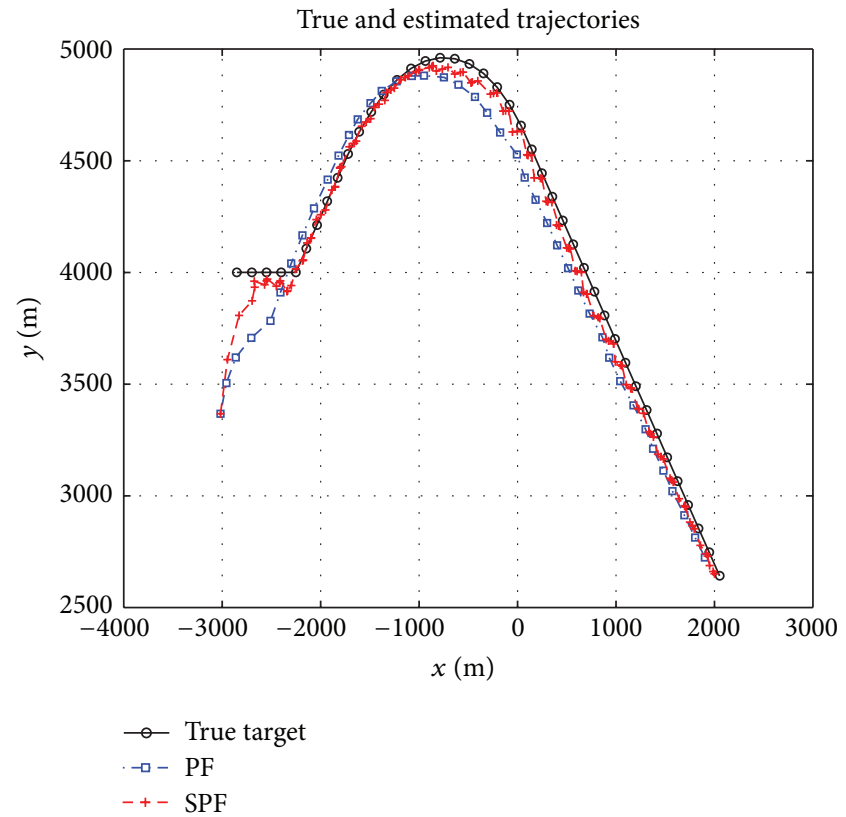

FIGURE 12: Tracking performance for a maneuvering target (20 MC trials).

true target. In addition, the range errors of one trial and the root-mean-square error (RMSE) of twenty Monte Carlo (MC) trials are plotted in Figures 10 and 11. The plots indicate that SPF estimated location converged to the true target location much faster, especially during the first few seconds. The range RMSEs of SPF and PF estimates at the 5th, 10th, and 15th seconds are listed in Table 3. Note that the difference between the RMSE values of the two tracking methods 


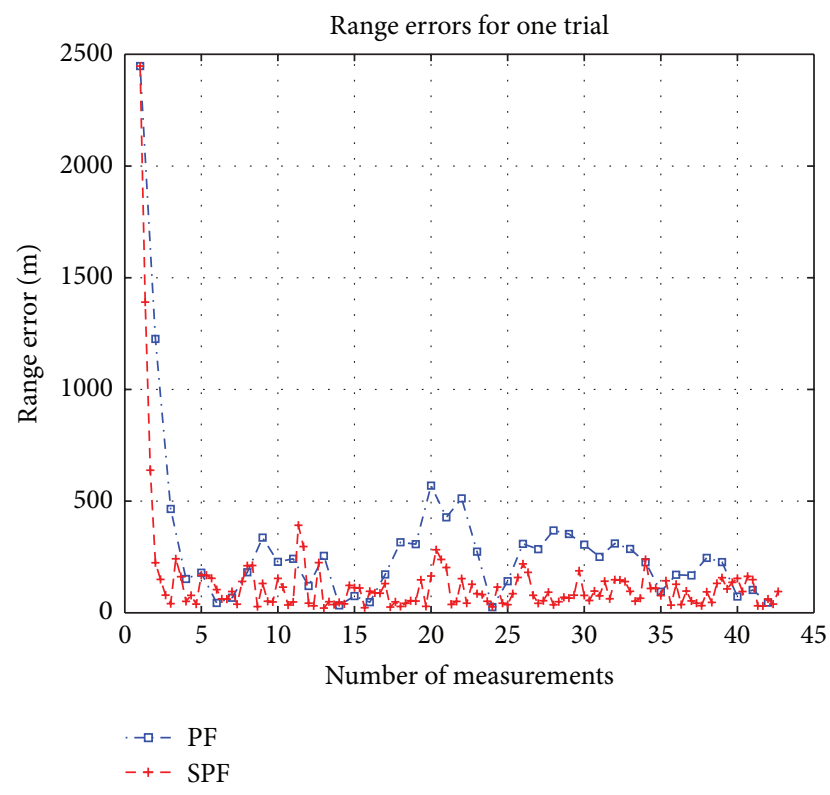

FIGURE 13: Range errors during each iteration (one trial).

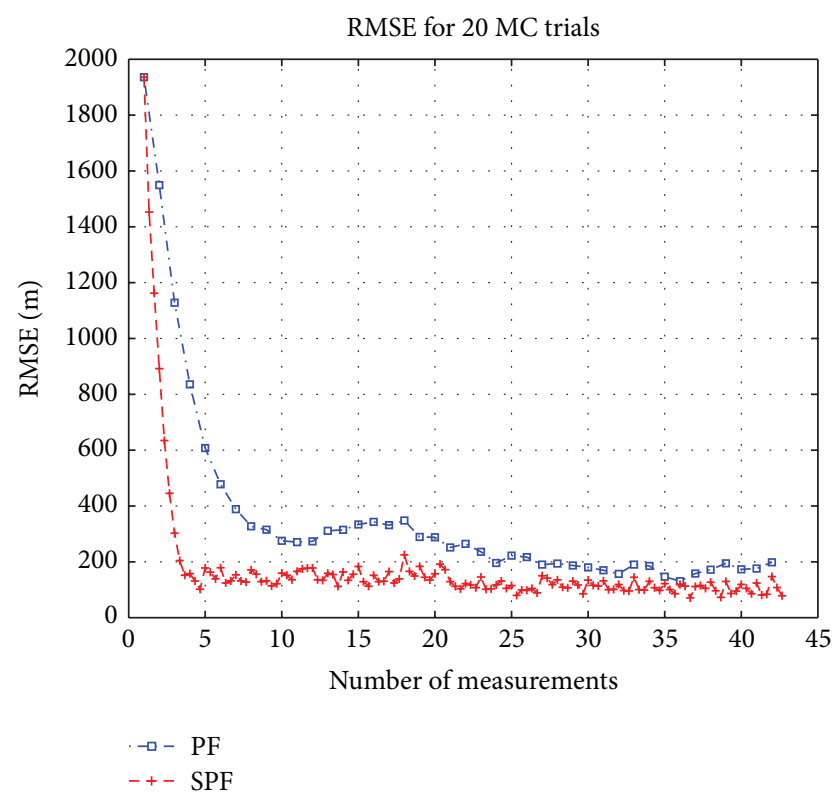

FIGURE 14: RMSE for a maneuvering target (20 MC trials).

increases during the first few measurements, because each new SPF estimate intelligently exploits prior estimates closer to the true target location which is not done when using PF. After convergence, SPF also performs better than PF in terms of RMSE.

In the following simulation, the benefit of the additive positional uncertainty will be shown through tracking a maneuvering target even though the motion model is unknown. For a noncooperative and maneuvering target $5 \mathrm{~km}$ away, Figure 12 shows that both PF and SPF are capable to track the target with decent accuracy. Again, $20 \mathrm{MC}$ trials are performed, and the range error for each iteration and the

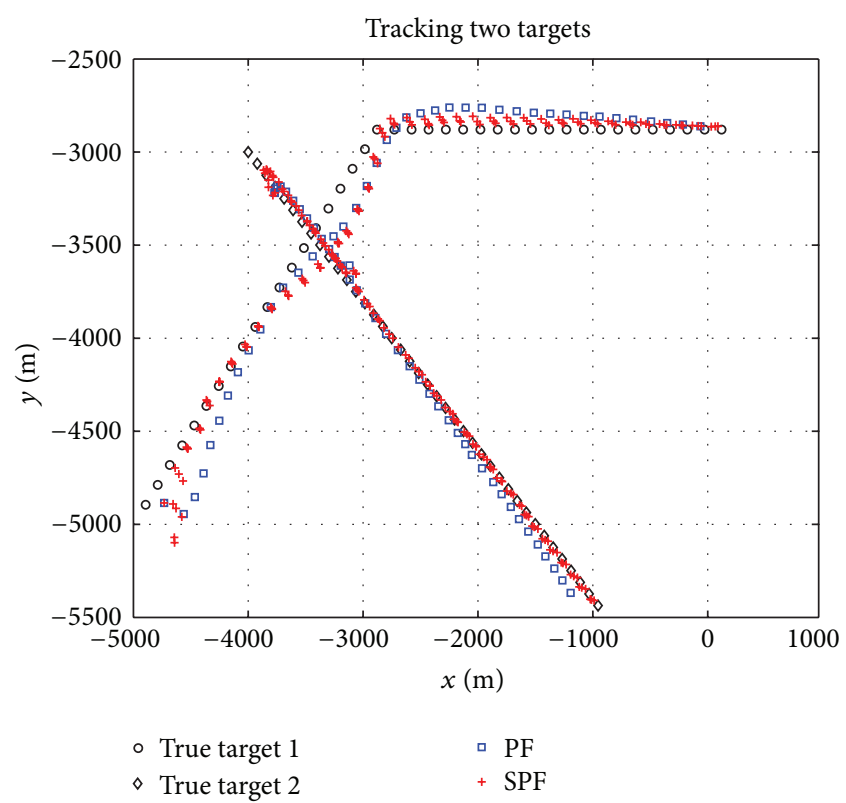

FIGURE 15: Tracking performance for multiple targets (20 MC trials).

TABLE 3: RMSE comparison (20 MC trials).

\begin{tabular}{lccc}
\hline & $\begin{array}{c}\text { RMSE after } \\
5 \text { seconds }\end{array}$ & $\begin{array}{c}\text { RMSE after } \\
10 \text { seconds }\end{array}$ & $\begin{array}{c}\text { RMSE after } \\
15 \text { seconds }\end{array}$ \\
\hline PF & $885.2 \mathrm{~m}$ & $488.7 \mathrm{~m}$ & $289.6 \mathrm{~m}$ \\
SPF & $212.9 \mathrm{~m}$ & $146.5 \mathrm{~m}$ & $45.33 \mathrm{~m}$ \\
\hline
\end{tabular}

RMSE results are depicted in Figures 13 and 14, respectively. Compared to the target at $10 \mathrm{~km}$ away in the first example, the error in the position estimate rolls off much faster when the target is closer to the sensors. Nevertheless, the improvement of SPF over PF is still noticeable in terms of convergence rate and estimation accuracy.

A multitarget scenario is simulated as well to test the capability to track multiple surrounding targets simultaneously. Since this paper focuses on the noncooperative targets, we assume two noncooperative targets located relative to own aircraft at $-4000 \mathrm{~m},-3000 \mathrm{~m}$ and $-5000 \mathrm{~m},-5000 \mathrm{~m}$, respectively. The relative speeds are $100 \mathrm{~m} / \mathrm{s}$ and $150 \mathrm{~m} / \mathrm{s}$, and the trajectories are drawn in Figure 15. Even though the two target trajectories cross over, the proposed method is still able to provide satisfactory estimated trajectories. However, similar to PSR, the target identification is not included in the radar report and trajectory, for each aircraft needs to be constructed using data association methods, which is another discrete problem.

\section{Conclusions}

The ADS-B system is considered as a cooperative surveillance technique to improve situational awareness while the ADS$B$ radar system is an innovative add-on implementation that exploits the standard ADS-B system with only a few additional integrated devices for the detection of noncooperative targets. It is significant to point out the independence of 
the communication and radar capability, because the radar performance will not be deteriorated in the event of an incorrect ADS-B message. The communication message is encoded using PPM while the radar signature is embedded in the sequence of the random phase modulation.

The ADS-B radar signal and system design are discussed thoroughly in this paper, and computer simulation demonstrates that the proposed approach allows the ADS-B radar system to track multiple noncooperative targets simultaneously. This research work opens a lot of opportunities for the ADS-B system to incorporate airborne sense-and-avoid capability, which can be useful for the purpose of collision avoidance for resource-limited UAVs [38, 39]. Moreover, the radar functionality of the ADS-B radar system has the potential to serve as a backup surveillance in the event of loss of GNSS function and make the communication system spoof resistant to incorrect ADS-B reports.

\section{References}

[1] SESAR Consortium, The ATM Target Concept: D3, Document Number DLM-0612-001-02-00, 108 pages, 2007, http://www .eurocontrol.int/sesar/gallery/content/public/docs/DLM-0612001-02-00.pdf.

[2] Joint Planning and Development Office, Concept of Operations for the Next Generation Air Transportation System, Version 3.2, 153 pages, 2010, http://jpe.jpdo.gov/ee/docs/conops/NextGen ConOps_v3_2.pdf.

[3] Study Group for the Future Air Traffic Systems, Collaborative Actions for Renovation of Air Traffic Systems (CARATS): Longterm Vision for the Future Air Traffic Systems, 56 pages, 2010, http://www.mlit.go.jp/common/000128185.pdf.

[4] P. Brooker, "SESAR and NextGen: investing in new paradigms," Journal of Navigation, vol. 61, no. 2, pp. 195-208, 2008.

[5] G. Wright, "NAV Canada implements ADS-B," in Proceedings of the Integrated Communications, Navigation and Surveillance Conference (ICNS '09), pp. 1-9, Arlington, Va, USA, 2009.

[6] L. Purton, H. Abbass, and S. Alam, "Identification of ADS-B system vulnerabilities and threats," in Proceedings of the 33rd Australasian Transport Research Forum (ATRF '10), pp. 1-16, Canberra, Australia, September 2010, http://www.patrec.org/ web_docs/atrf/papers/2010/1916_222\%20-\%20Purton\%20Abbass\%20Alam.pdf.

[7] J. A. Besada, J. Garcia, G. de Miguel, F. J. Jimenez, G. Gavin, and J. R. Casar, "Data fusion algorithms based on radar and ADS measurements for ATC application," in Proceedings of the IEEE International Radar Conference, pp. 98-103, Alexandria, Va, USA, May 2000.

[8] J. L. R. Da Silva, J. F. B. Brancalion, and D. Fernandes, "Data fusion techniques applied to scenarios including ADS-B and radar sensors for air traffic control," in Proceedings of the 12th International Conference on Information Fusion (FUSION '09), pp. 1481-1488, Seattle, Wash, USA, July 2009.

[9] C. Mensing, S. Sand, and A. Dammann, "Hybrid data fusion and tracking for positioning with GNSS and 3GPP-LTE," International Journal of Navigation and Observation, vol. 2010, Article ID 812945, 12 pages, 2010.

[10] A. Grant, P. Williams, N. Ward, and S. Basker, "GPS jamming and the impact on maritime navigation," Journal of Navigation, vol. 62 , no. 2, pp. 173-187, 2009.
[11] Y. Zhang and J. Qiao, “ADS-B radar system,” US Patent 7414567, Intelligent Automation, Rockville, Md, USA, 2008.

[12] M.-S. Huang, R. M. Narayanan, and A. Feinberg, "Multiple targets estimation and tracking for ADS-B radar system," in Proceedings of the IEEE/AIAA 27th Digital Avionics Systems Conference (DASC '08), pp. 3C11-3C110, St. Paul, Minn, USA, 2008.

[13] C. P. Lai, Y. J. Ren, and C. Lin, "ADS-B based collision avoidance radar for unmanned aerial vehicles," in 2009 IEEE MTTS International Microwave Symposium (IMS '09), pp. 85-88, Boston, Mass, USA, June 2009.

[14] S. Wang, Y. Zhang, A. Huston, Z. Li, M. Mallo, and J. Fagan, "Phase modulated waveforms for transponder-based radar sensing: signal optimization and experiments," IEEE Transactions on Aerospace and Electronic Systems, vol. 47, no. 3, pp.17331753, 2011.

[15] M.-S. Huang and R. M. Narayanan, "Non-cooperative collision avoidance concept for Unmanned Aircraft System using satellite-based radar and radio communication," in Proceedings of the 30th Digital Avionics Systems Conference-Closing the Generation Gap: Increasing Capability for Flight Operations among Legacy, Modern and Uninhabited Aircraft (DASC '11), pp. 5C21-5C29, Seattle, Wash, USA, 2011.

[16] R. Barhydt and A. W. Warren, "Development of intent information changes to revised minimum aviation system performance standards for automatic dependent surveillance broadcast (RTCA/DO-242A)," NASA Technical Memorandum TM 2002-211669, 2002.

[17] K. Owusu and G. Dunstone, "Development of cockpit display of traffic information (CDTI)," in Proceedings of the 3rd Meeting of Automatic Dependent Surveillance-Broadcast (ADS-B) Study and Implementation Task Force (ADS-B TF '05), Bangkok, Thailand, March 2005.

[18] S. R. J. Axelsson, "Noise radar using random phase and frequency modulation," IEEE Transactions on Geoscience and Remote Sensing, vol. 42, no. 11, pp. 2370-2384, 2004.

[19] R. O. Schmidt, "Multiple emitter location and signal parameter estimation," IEEE Transactions on Antennas and Propagation, vol. 34, no. 3, pp. 276-280, 1986.

[20] T. Akiyama, T. Yamaoka, and N. Hamada, "2-D angle estimation using the constrained MUSIC with circular array," in Proceedings of the 5th International Symposium on Signal Processing and Its Applications, pp. 877-880, Brisbane, Australia, August 1999.

[21] Z. Saidi and S. Bourennane, "Cumulant-based coherent signal subspace method for bearing and range estimation," EURASIP Journal on Advances in Signal Processing, vol. 2007, Article ID 84576, 2007.

[22] H. Hung and M. Kaveh, "Focussing matrices for coherent signal-subspace processing," IEEE Transactions on Acoustics, Speech, and Signal Processing, vol. 36, no. 8, pp. 1272-1281, 1988.

[23] P. Fearnhead, Sequential Monte Carlo methods in filter theory [Ph.D. thesis], University of Oxford, Oxford, UK, 1998.

[24] Y. Bar-Shalom and T. E. Fortmann, Tracking and Data Association, Academic Press, New York, NY, USA, 1988.

[25] I. Hwang, H. Balakrishnan, K. Roy, and C. Tomlin, "Multipletarget tracking and identity management with application to aircraft tracking," Journal of Guidance, Control, and Dynamics, vol. 30, no. 3, pp. 641-653, 2007.

[26] F. Gustafsson, F. Gunnarsson, N. Bergman et al., "Particle filters for positioning, navigation, and tracking," IEEE Transactions on Signal Processing, vol. 50, no. 2, pp. 425-437, 2002. 
[27] M. S. Arulampalam, S. Maskell, N. Gordon, and T. Clapp, "A tutorial on particle filters for online nonlinear/non-Gaussian Bayesian tracking," IEEE Transactions on Signal Processing, vol. 50, no. 2, pp. 174-188, 2002.

[28] B. Ristic, S. Arulampalam, and N. Gordon, Beyond the Kalman Filter: Particle Filters for Tracking Applications, Artech House, Norwood, Mass, USA, 2004.

[29] N. Gordon, D. Salmond, and C. Ewing, "Bayesian state estimation for tracking and guidance using the bootstrap filter," Journal of Guidance, Control, and Dynamics, vol. 18, no. 6, pp. 1434-1443, 1995.

[30] M. Isard and A. Blake, "CONDENSATION—conditional density propagation for visual tracking," International Journal of Computer Vision, vol. 29, no. 1, pp. 5-28, 1998.

[31] N. J. Gordon, D. J. Salmond, and A. F. M. Smith, "Novel approach to nonlinear/non-gaussian Bayesian state estimation," IEE Proceedings, Part F, vol. 140, no. 2, pp. 107-113, 1993.

[32] E. Mazor, A. Averbuch, Y. Bar-Shalom, and J. Dayan, "Interacting multiple model methods in target tracking: a survey," IEEE Transactions on Aerospace and Electronic Systems, vol. 34, no. 1, pp. 103-123, 1998.

[33] Z. Khan, T. Balch, and F. Dellaert, "An MCMC-based particle filter for tracking multiple interacting targets," in Proceedings of the 8th European Conference on Computer Vision (ECCV '04), vol. 3024 of Lecture Notes in Computer Science, pp. 279-290, 2004.

[34] H. A. P. Blom, "An efficient filter for abruptly changing systems," in Proceedings of the 23rd IEEE Conference on Decision and Control, pp. 656-658, Las Vegas, Nev, USA, December 1984.

[35] J. K. Tugnait, "Detection and estimation for abruptly changing systems," in Proceedings of the 20th IEEE Conference on Decision and Control, pp. 1357-1362, San Diego, Calif, USA, December 1981.

[36] J. S. Liu, "Metropolized independent sampling with comparisons to rejection sampling and importance sampling," Statistics and Computing, vol. 6, no. 2, pp. 113-119, 1996.

[37] A. Kong, J. S. Liu, and W. H. Wong, "Sequential imputations and Bayesian missing data problems," Journal of the American Statistical Association, vol. 89, no. 425, pp. 278-288, 1994.

[38] B. A. Kumar and D. Ghose, "Radar-assisted collision avoidance/guidance strategy for planar flight," IEEE Transactions on Aerospace and Electronic Systems, vol. 37, no. 1, pp. 77-90, 2001.

[39] S. C. Han, H. Bang, and C. S. Yoo, "Proportional navigationbased collision avoidance for UAVs," International Journal of Control, Automation and Systems, vol. 7, no. 4, pp. 553-565, 2009. 

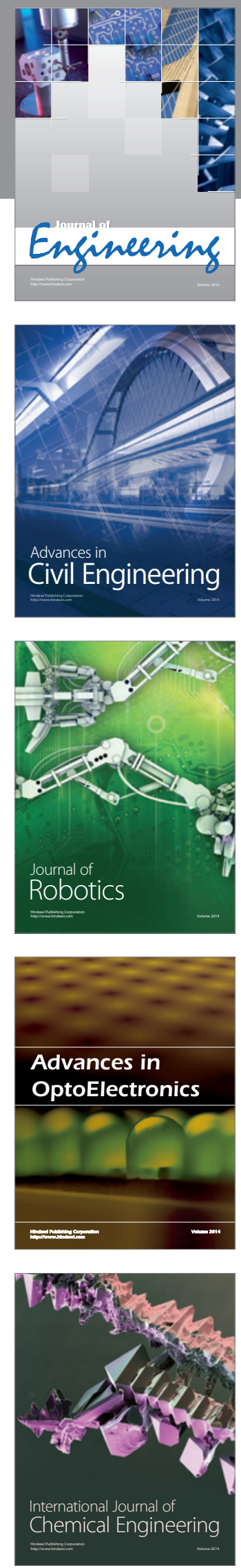

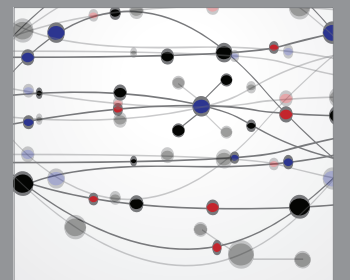

The Scientific World Journal
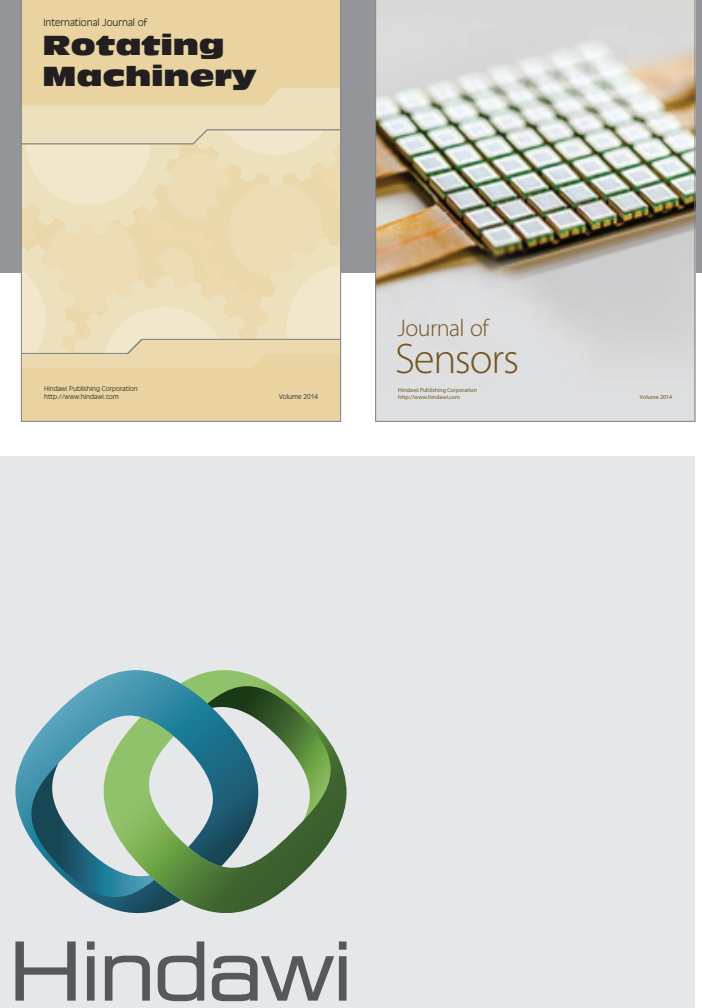

Submit your manuscripts at http://www.hindawi.com
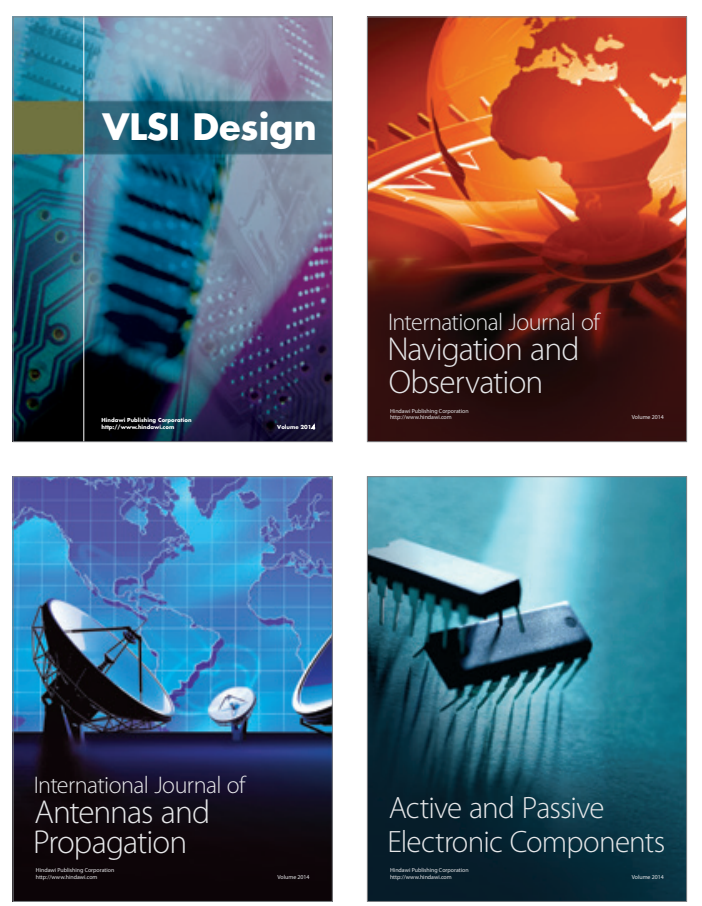
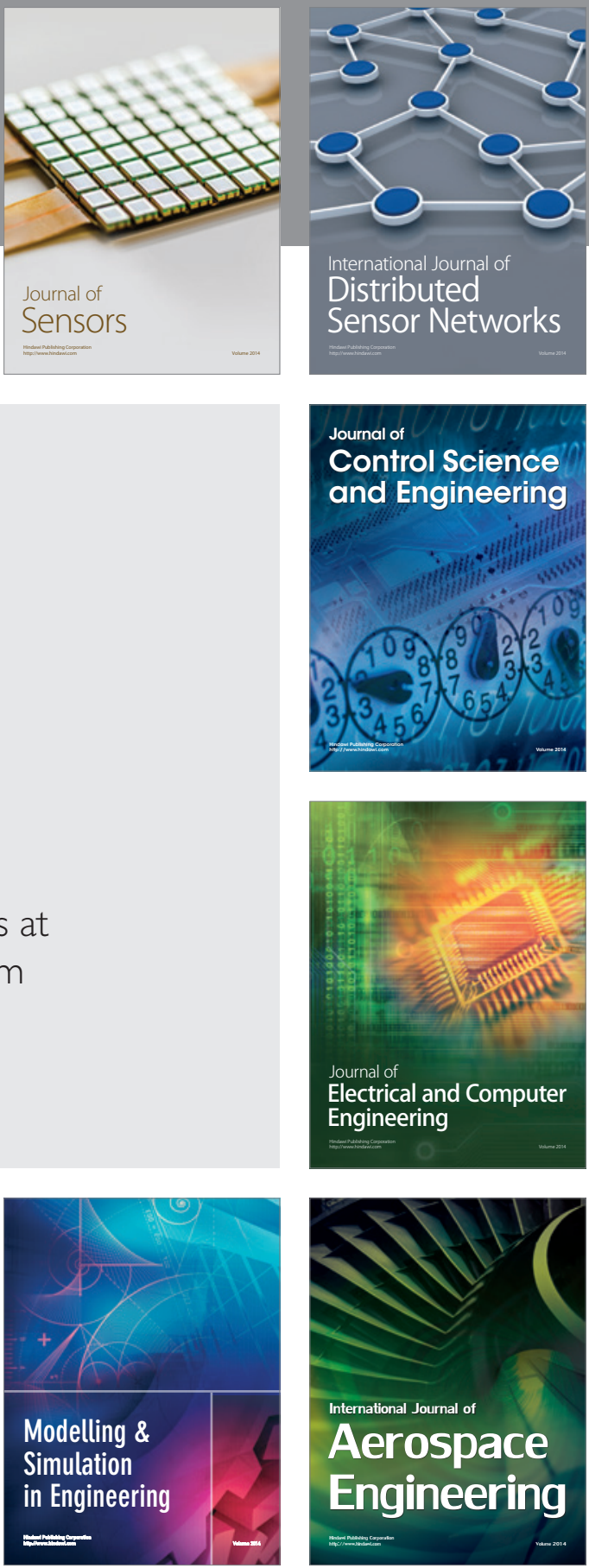

Journal of

Control Science

and Engineering
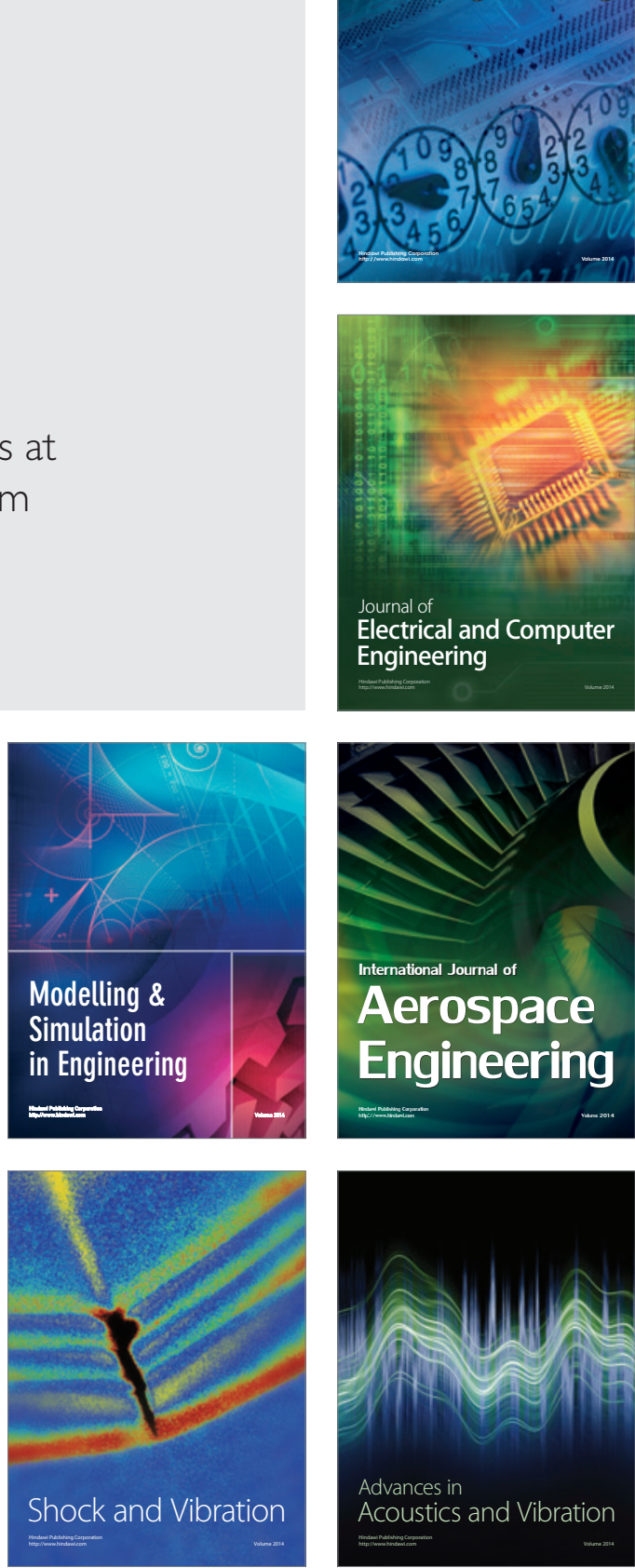